Special Issue of the 6th International Congress \& Exhibition (APMAS2016), Maslak, Istanbul, Turkey, June 1-3, 2016

\title{
Green Synthesis of Pd Nanoparticles via Gallic Acid
}

\begin{abstract}
M. CAN*
Vocational High School of Arifiye and Department of Chemistry, Sakarya University, 54187 Sakarya, Turkey

A facile, eco-friendly, room-temperature method for rapid one-pot synthesis of Pd metallic nanoparticles has been developed based on the successive reduction of $\mathrm{Pd}(\mathrm{II})$ precursors with gallic acid in an aqueous environment. The morphology and surface analysis of the resulting Pd nanoparticles were confirmed by aberration corrected scanning transmission electron microscopy followed by X-ray photoelectron spectroscopy. The particle size of palladium nanoparticles was calculated as $16 \mathrm{~nm}$. This study demonstrates that the synthesis of nanoparticles (reduction and stabilization) can be effectively achieved using gallic acid.
\end{abstract}

DOI: 10.12693/APhysPolA.131.569

PACS/topics: $82.30 . \mathrm{Hk}$

\section{Introduction}

Synthesis of metal nanoparticles and nanocrystals with manipulated morphology and size has drawn considerable attention in the past decades because they present a useful platform to construct new materials in which diversified functions can be tailored at the atomic scale, and provide a tremendous potential for various applications [1]. Among them, fabrication of palladium nanoparticles (PdNPs) is of great importance and fundamental merit to the scientific community due to their low toxicity, biocompatibility, antimicrobial activity, optical and electrochemical properties [2-4]. As most of physiochemical characteristics of PdNPs highly depend on their particle properties that allow their unique functionalities, it is important from a practical point of view to develop synthetic protocols to fabricate PdNPs with a controllable size, size distribution and crystalline morphology. The aim of this study is to understand changes of particle size with changing molar ratio of $\mathrm{GA} / \mathrm{Pd}^{2+}$. To this end, a series of $\mathrm{Pd}^{2+}$ mixtures with different molar ratios were blended with GA solution at room temperature under ambient atmosphere. Much to our delight, PdNPs could be easily synthesized under mild conditions using GA, a typical natural non-toxic polyphenol, as the reductant and stabilizer, which is much more environmentally benign than the conventional 'bottom-up' methods of nanoparticle preparation, that need toxic chemical reducing agents and/or stabilizers. Consequently, our investigations have provided the first successful example of a "green" synthesis of Pd nanoparticles by using gallic acid as the reductant and stabilizer.

\section{Experimental}

Gallic acid (GA) was purchased from Alfa Aesar $\mathrm{GmbH} \&$ Co. $\mathrm{PdCl}_{3} \cdot 3 \mathrm{H}_{2} \mathrm{O}, \mathrm{NH}_{3}, \mathrm{HCOH}, \mathrm{HNO}_{3}, \mathrm{HCl}$, $\mathrm{NaCl}$ and $\mathrm{NaOH}$ were purchased from Merck Company.

\footnotetext{
*e-mail: mustafacan@sakarya.edu.tr
}

AAS standard solutions for determination of PGM were purchased form UltraScientific Company. All other reagents were analytical grade. The aqueous $\mathrm{Pd}(\mathrm{II})$ stock solution was prepared from solid $\mathrm{PdCl}_{2} .2 \mathrm{mg} \mathrm{Pd}^{2+}$ were added into $50 \mathrm{ml}$ of $\mathrm{GA}$ solution $(\mathrm{GA}=15,25,50,100$, $200,400,600,800$, and $1000 \mathrm{mg}$ ) under constant stirring at room temperature, which allowed the preparation of a homogenous GA-PdNP colloid. pH-controlled solutions of $\mathrm{Pd}(\mathrm{II})$ were obtained by dilution with $\mathrm{NaOH}$ or $\mathrm{HNO}_{3}$ to adjust the $\mathrm{H}^{+}$concentration to the desired value. This way the effect of the $\mathrm{pH}$ was investigated. Temperature experiments were conducted at $25^{\circ} \mathrm{C}, 40^{\circ} \mathrm{C}$, and $60^{\circ} \mathrm{C}$, respectively.

The FTIR spectrum of samples was analyzed using Pelkin Elmer Spectrum One instrument. TEM images of GA-PdNPs colloids were taken on a Jeol Jem 1220 Transmission Electron Microscope (TEM). X-ray photoelectron spectra (XPS) of the samples were recorded on a SPECS EA 300.

\section{Results and discussion}

Gallic acid contains an aromatic ring, bearing a carboxyl group, and three adjacent hydroxyl groups (Fig. 1). These phenolic hydroxyls are able to chelate with metal ions forming five-membered chelate rings, and can also be easily oxidized to the corresponding benzoquinones, when exposed to air or if they come into contact with electrophilic ions [5], which suggests that GA can be used as an effective reducing agent. On the other hand, $\mathrm{Pd}^{2+}$ has high oxidation-reduction potential, which suggests that $\mathrm{Pd}(\mathrm{II})$ ions may play a role as oxidants when they come into contact with GA. Thus we believe that GA could serve as a reducing agent for the reduction of $\mathrm{Pd}^{2+}$ to form PdNPs. With the use of GA, the synthesis of PdNP colloids is so simple that no surfactant, photo-irradiation, or heat treatment is required $[6,7]$. The preparation of GA-PdNP colloids is so efficient that the synthetic process can be completed within $5 \mathrm{~min}$, which shows great practical value for the large-scale preparation of PdNP colloids. Additionally, the synthetic procedure for GAx-PdNPs preparation showed high reproducibility. In most cases, the color of the mixture 
quickly changed to brown after the $\mathrm{Pd}(\mathrm{II})$ stock solution and GA were mixed together. However, the color of the specimen having low concentration of GA (25 mg) remained yellow even after $24 \mathrm{~h}$.
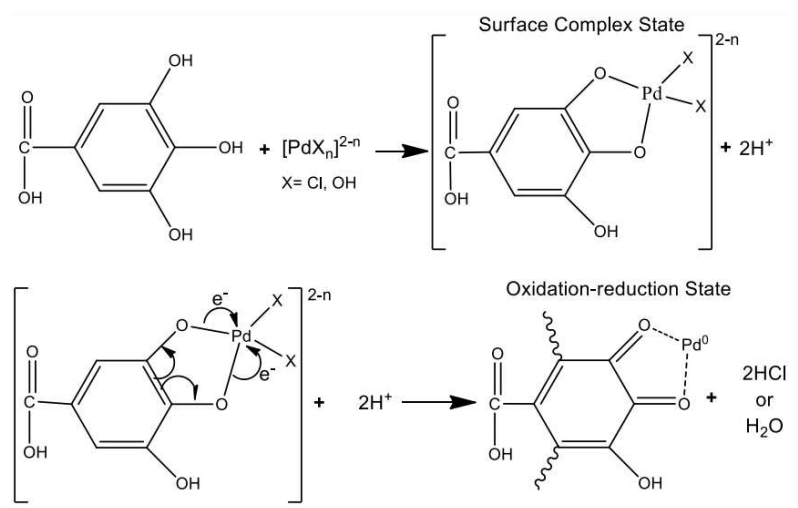

Fig. 1. Molecular structure of GA and its interaction with $\mathrm{Pd}(\mathrm{II})$ ions.

Figure 2 shows the TEM image of $\mathrm{GA}_{50}-\mathrm{PdNPs}$ and the corresponding histogram of particle size distribution. The mean particle diameter of $\mathrm{GA}_{50}-\mathrm{PdNPs}$ is $23 \mathrm{~nm}$, and no agglomeration of PdNPs was observed. Hence, GA is able to reduce Pd (II) to form PdNPs and serve as a stabilizing agent to prevent agglomeration of PdNPs.

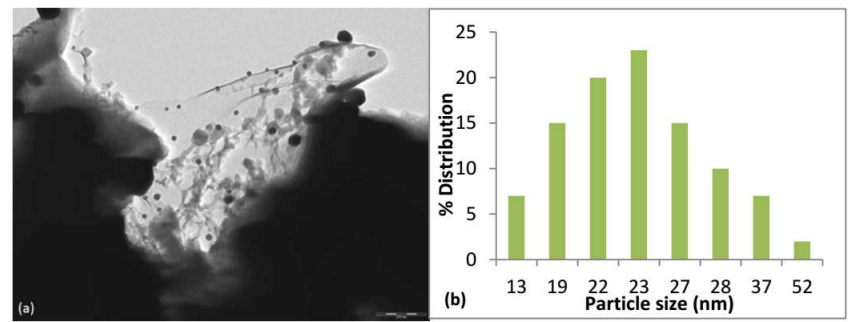

Fig. 2. TEM images of $\mathrm{GA}_{50}-\mathrm{PdNPs}$ (a) and particle size distribution (b).

Figure 3 shows general XPS spectra of $\mathrm{GA}_{50}-\mathrm{PdNPs}$ colloid. As can be seen, main components, such as Pd, $\mathrm{C}$ and $\mathrm{O}$ atoms, have a strong adsorption density. Three major peaks corresponding to binding energy of 335, 337, and $340 \mathrm{eV}$ are related to $\mathrm{Pd} 3 \mathrm{~d} 5 / 2$ and $\mathrm{Pd} 3 \mathrm{~d} 3 / 2$ [8]. It has been observed that $\mathrm{Pd}$ is present predominantly in the $\mathrm{Pd}^{0}$ state $(335 \mathrm{eV}$ and $340 \mathrm{eV})$, together with little $\mathrm{Pd}^{2+}(337 \mathrm{eV})$.

\section{Conclusions}

This study describes an extremely simple and effective approach for the preparation of PdNPs colloids. In this method, GA serves as a reducing agent as well as a stabilizing agent. It was found that GA was effective in reducing $\mathrm{Pd}^{2+}$ to form $\mathrm{PdNPs}$ at room temperature without any additional reagent or treatment. The mean diameter

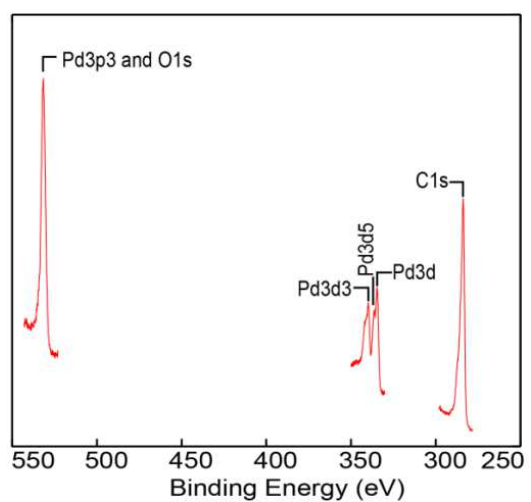

Fig. 3. XPS general spectra of the $\mathrm{GA}_{50}-\mathrm{PdNPs}$

and size distribution of PdNPs can be facilely controlled by varying the concentration of GA. GA is biocompatible, which increases the biocompatibility of PdNPs. Furthermore, this green approach for the synthesis of PdNPs is extremely efficient and highly reproducible, which shows great potential for practical applications.

\section{Acknowledgments}

This work is supported by the Scientific and Technological Research Council of Turkey (TUBITAK) under the contract number $115 \mathrm{Z} 054$

\section{References}

[1] M. Can, Res. J. Chem. Environ. 17, 1 (2013).

[2] M. Can, Res. J. Chem. Environ. 17, 117 (2013).

[3] M.M. Kumari, S.A. Aromal, D. Philip, Spectrochim. Acta A 103, 130 (2013).

[4] Y.-L. Ko, S. Krishnamurthy, Y.-S. Yun, J. Nanosci. Nanotechnol. 15, 412 (2015).

[5] M. Can, E. Bulut, M. Ozacar, Ind. Eng. Chem. Res. 51, $6052(2012)$.

[6] X. Huang, H. Wu, X. Liao, B. Shi, Green Chem. 12, 395 (2010).

[7] Y.W. Lee, M. Kim, Z.H. Kim, S.W. Han, J. Am. Chem. Soc. 131, 17036 (2009).

[8] H. Saikia, B.J. Borah, Y. Yamada, P. Bharali, J. Colloid Interface Sci. 486, 46 (2017). 